\title{
Decay of Loschmidt Echo Enhanced by Quantum Criticality
}

\author{
H.T. Quan ${ }^{1}$, Z. Song ${ }^{2}$, X.F. Liu ${ }^{3}$, P. Zanardi ${ }^{4}$ and C.P. Sun ${ }^{1,2 *}$ \\ 1 Institute of Theoretical Physics, Chinese Academy of Sciences, Beijing, 100080, China \\ ${ }^{2}$ Department of Physics, Nankai University, Tianjin 300071, China \\ 3 Department of Mathematics, Peking University, Beijing, 100871, China and \\ ${ }^{4}$ Institute for Scientific Interchange Foundation Villa Gualino Viale Settimio Severo 65 I-10133 Torino, Italy
}

\begin{abstract}
We study the transition of a quantum system $S$ from a pure state to a mixed one, which is induced by the quantum criticality of the surrounding system $E$ coupled to it. To characterize this transition quantitatively, we carefully examine the behavior of the Loschmidt echo (LE) of $E$ modelled as an Ising model in a transverse field, which behaves as a measuring apparatus in quantum measurement. It is found that the quantum critical behavior of $E$ strongly affects its capability of enhancing the decay of LE: near the critical value of the transverse field entailing the happening of quantum phase transition, the off-diagonal elements of the reduced density matrix describing $S$ vanish sharply.
\end{abstract}

PACS numbers: 03.65.Yz, 05.70.Jk, 03.65.Ta, 05.50.+q,

Introduction: Nowadays quantum -classical transitions described by a reduction from a pure state to a mixture [1, 2] renew interests in many areas of physics, mainly due to the importance of quantum measurement and decoherence problem in quantum computing. To study this transition, some exactly-solvable models were proposed for a system coupled to the macroscopic $3,4,5$, or classical 6, 7] surrounding systems. Relevantly, in association with the quantum -classical transition in quantum chaos, the concept of Loschmidt echo (LE) from NMR experiments was introduced to describe the hypersensitivity of the time evolution to the perturbations experienced by the surrounding system $[8,9]$. In this letter, by a concrete example, we will show how quantum phase transition (QPT) [10] of the surrounding system can also sensitively affect the decay of its own LE, which means a dynamic reduction of its coupled system from pure state to a mixed one. Here, we note that a QPT effect has been explored for the Dicke model at the transition from quasi-integrable to quantum chaotic phases [1].

As a quantum critical phenomenon, QPT happens at zero temperature, at which the thermal fluctuations vanish. Thus QPT is driven only by quantum fluctuation, and the uncertainty relation lie at the heart of various QPT phenomena. On the other hand, the randomness of the relative phase, which causes pure-mixed state transition, also has its source in the uncertainty principle 12. It is this observation that enlightens us to explore the relationship between QPT and the pure-mixed state transition described dynamically by the time evolution of the LE. It is common that the ground state of the critical system is very sensitive to the varying magnitude of the coupling constant [13], or the system experiences a spontaneous symmetry breaking at the critical point. Up to now, all of the known models of QPT possess this property. Actually, this kind of critical sensitiveness can be well understood by resorting to the concepts of quantum chaos through the LE [9] or macroscopic enhancement of phase randomness [12.
Our approach is based on the Hepp-Coleman (HC) model [3, 4], which was initially proposed as a model for quantum measurement. In our generalization, the free spin $1 / 2$ ensemble, as a model of measuring apparatus, is replaced bv the Ising spin chain $E$ in a transverse field.

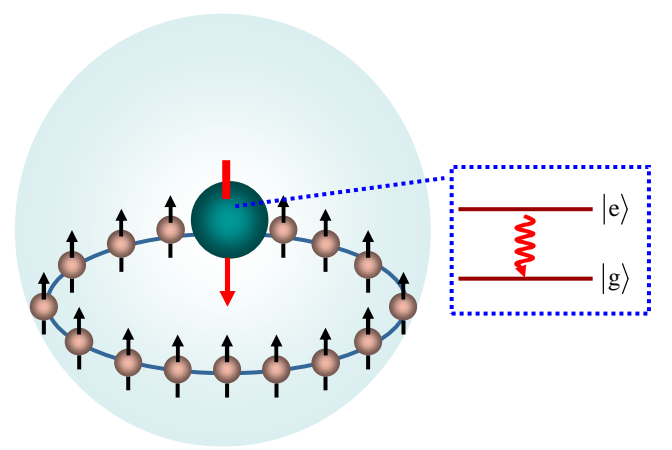

FIG. 1: (Color online) A schematic diagram of the physical implementation of the generalized Hepp-Coleman Model. The spins are arranged in a circle to form a ring array $E$. The central two-level system $S$ possesses homogeneous couplings due to the overlaps of symmetric spacial wave function of $S$ with those of spins.

Model setup based on quantum phase transition: Our model concerning the decay of LE, illustrated in Fig. 1, is very similar to the Hepp-Coleman model [3, 4] or its generalizations [5, 6, 7]. We take the surrounding system $E$ to be an Ising spin chain in a transverse field, which 
satisfies the Born-Von Karman condition automatically. Consider a two level system $S$ with the excited state $|e\rangle$ and the ground state $|g\rangle$, which is transversely coupled to $E$. The corresponding Hamiltonian reads as follows:

$$
H(\lambda, \delta)=-J \sum_{j}\left(\sigma_{j}^{z} \sigma_{j+1}^{z}+\lambda \sigma_{j}^{x}+\delta|e\rangle\langle e| \sigma_{j}^{x}\right),
$$

where $J$ and $\lambda$ characterize the strengths of the Ising interaction and the coupling to the transverse field respectively; $\delta$ indicates the small perturbation coupling of $S$ to $E ; \sigma_{i}^{\alpha}(\alpha=x, y, z)$ are the Pauli operators defined on the $i$ th site in the lattice with lattice spacing $a$.

We assume the two-level system is initially in a superposition state $\left|\phi_{s}(0)\right\rangle=c_{g}|g\rangle+c_{e}|e\rangle$, where the coefficients $c_{g}$ and $c_{e}$ satisfy $\left|c_{g}\right|^{2}+\left|c_{e}\right|^{2}=1$. Then the evolution of the Ising spin chain initially prepared in $|\varphi(0)\rangle$, will split into two branches $\left|\varphi_{\alpha}(t)\right\rangle=\exp \left(-i H_{\alpha} t\right)|\varphi(0)\rangle$ $(\alpha=e, g)$, and the total wave function is obtained as $|\psi(t)\rangle=c_{g}|g\rangle \otimes\left|\varphi_{g}(t)\right\rangle+c_{e}|e\rangle \otimes\left|\varphi_{e}(t)\right\rangle$. Here, the evolutions of the two branch wave functions $\left|\varphi_{\alpha}(t)\right\rangle$ are driven respectively by the two effective Hamiltonians $H_{g}=H(\lambda, 0)$ and $H_{e}=H(\lambda, \delta) \equiv H_{g}+V_{e}$. Obviously, both $H_{g}$ and $H_{e}$ describe the Ising model in a transverse field, but with a tiny difference in the field strength. The central quantum system in two different states $|e\rangle$ and $|g\rangle$ will exert slightly different back actions on the surrounding system, which manifest as two effective potentials $V_{e}=-J \delta \sum_{j} \sigma_{j}^{x}$ and $V_{g}=0$. This difference just results in the decay of the LE [9] defined as

$$
L(\lambda, t)=\left|\left\langle\varphi_{g}(t) \mid \varphi_{e}(t)\right\rangle\right|^{2} .
$$

To show the key role of LE in depicting quantum decoherence of the central system, and manifest the difference between LE and decoherence, we define the purity [15] $P=\operatorname{Tr}_{S}\left(\rho_{S}^{2}\right)=\operatorname{Tr}_{S}\left\{\left[\operatorname{Tr}_{E} \rho(t)\right]^{2}\right\}$ to describe the decoherence. Here, $\rho(t)=|\psi(t)\rangle\langle\psi(t)|$, and $\operatorname{Tr}_{\alpha}$ means tracing over the variable of $\alpha,(\alpha=E, S)$. A straightforward calculation reveals the relationship between LE and the purity as $P=1-2\left|c_{e} c_{g}\right|^{2}[1-L(\lambda, t)]$. This equation indicates that the purity depends on the initial states of the central system and the surrounding system $E$, but the LE only depends on the initial state of $E$. For simplicity, we assume the surrounding system is initially prepared in the ground state. In the following discussion, we will focus on the decay problem of LE induced by the coupling with the central system.

Exact solution for the Loschmidt echo: We now prepare to prove that, just at the critical point $\lambda=\lambda_{c}=1$, the decay of LE is enhanced, accompanied by the QPT in one of the two evolution branches.

We first diagonalize the effective Hamiltonian as $H_{e}=$ $\sum_{k} \varepsilon_{e}^{k}\left(A_{k}^{\dagger} A_{k}-1 / 2\right)$ in terms of the normal mode operators [10, 14]

$$
A_{k}=\sum_{l} \frac{e^{-i k a l}}{\sqrt{N}} \prod_{s<l} \sigma_{s}^{[x]}\left(u_{e}^{k} \sigma_{l}^{[+]}-i v_{e}^{k} \sigma_{l}^{[-]}\right),
$$

which satisfy the canonical fermion anti-commutation relations. Here, $N$ is the number of sites of the spin chain, and $\sigma_{l}^{[ \pm]}=\left(-\sigma_{l}^{z} \pm i \sigma_{l}^{y}\right) / 2$ is defined by the Pauli matrices $\sigma_{l}^{\alpha}, \alpha=x, y, z$. The coefficients $u_{e}^{k}=$ $\cos \left(\theta_{e}^{k} / 2\right), v_{e}^{k}=\sin \left(\theta_{e}^{k} / 2\right)$ depend on the the angle

$$
\theta_{e}^{k}=\theta_{e}^{k}(\delta)=\arctan \left[\frac{-\sin (k a)}{\cos (k a)-(\lambda+\delta)}\right]
$$

The corresponding single quasi-excitation energy $\varepsilon_{e}^{k}$ is

$$
\varepsilon_{e}^{k}(\delta)=2 J \sqrt{1+(\lambda+\delta)^{2}-2(\lambda+\delta) \cos (k a)} .
$$

Note that, in writing down the known result (3) in a compact form, we have combined the Jordan-Wigner map and the Fourier transformation to the momentum space [10, 14].

The effective Hamiltonian $H_{g}$ can be diagonalized in a similar way: $H_{g}=\sum_{k} \varepsilon_{g}^{k}\left(B_{k}^{\dagger} B_{k}-1 / 2\right)$. In this case the single quasi-excitation energy is $\varepsilon_{g}^{k}=\varepsilon_{e}^{k}(0)$ and the corresponding fermionic quasi-excitation operators $B_{k}$ can be obtained by the following Bogliubov transformation

$$
B_{ \pm k}=\cos \left(\alpha_{k}\right) A_{ \pm k}-i \sin \left(\alpha_{k}\right)\left(A_{\mp k}\right)^{\dagger} .
$$

Here, $\alpha_{k}=\left[\theta_{g}^{k}-\theta_{e}^{k}\right] / 2$, and $\theta_{g}^{k}$ are defined by $\theta_{g}^{k}=\theta_{e}^{k}(0)$.

We suppose that the spin chain is initially in the ground state $|\varphi(0)\rangle=|G\rangle_{g}$ of the Ising spin chain in a transverse field depicted by $H_{g}$, i.e., $B_{k}|G\rangle_{g}=0$ for any operator $B_{k}$. Then from Eq. ([6) the state $|G\rangle_{g}$ can be rewritten as a BCS-like state:

$$
|G\rangle_{g}=\prod_{k>0}\left[\cos \left(\alpha_{k}\right)-i \sin \left(\alpha_{k}\right) A_{k}^{\dagger} A_{-k}^{\dagger}\right]|G\rangle_{e},
$$

where $|G\rangle_{e}$ is the ground state of $H_{e}$. This explicit expression of $|G\rangle_{g}$ enables us to calculate straightforwardly the LE (2), which assumes the following factorized form:

$$
L(\lambda, t)=\prod_{k>0} F_{k}=\prod_{k>0}\left[1-\sin ^{2}\left(2 \alpha_{k}\right) \sin ^{2}\left(\varepsilon_{e}^{k} t\right)\right] .
$$

Quantum-classical transition at critical point of QPT: Since each factor $F_{k}$ in Eq (8) has a norm less than unity, we may well expect $L(\lambda, t)$ to decrease to zero in the large $N$ limit under some reasonable conditions. This kind of factorized structure was first discovered and systematically studied by one of the authors in developing the quantum measurement theory in classical or macroscopic limit 7] and it has been applied to analyze the universality of decoherence influence from environment on quantum computing [17]. Now we study in detail the critical behavior of the surrounding system near the critical point $\lambda_{c}=1$ and its relation to the sensitive evolution of the LE perturbed by the central system even for a finite $N$. This turns out to reveal a novel mechanism responsible for the enhanced decay of LE. 
Let us first make a heuristic analysis of the features of the LE. For a cut-off frequency $K_{c}$ we define the partial product for the LE

$$
L_{c}(\lambda, t) \equiv \prod_{k>0}^{K_{c}} F_{k} \geq L(\lambda, t),
$$

and the corresponding partial sum $S(\lambda, t)=\ln L_{c} \equiv$ $-\sum_{k>0}^{K_{c}}\left|\ln F_{k}\right|$. For small $k$ we have $\varepsilon_{e}^{k} \approx 2 J|1-\lambda-\delta|$, $\sin ^{2}\left[2 \alpha_{k}\right] \approx(\delta k a)^{2} /(1-\lambda)^{2}(1-\lambda-\delta)^{2}$. As a result, if $K_{c}$ is small enough we have

$$
S(\lambda, t) \approx-\frac{\delta^{2} E\left(K_{c}\right) \sin ^{2}(2 J t|1-\lambda-\delta|)}{(1-\lambda)^{2}(1-\lambda-\delta)^{2}}
$$

where $E\left(K_{c}\right)=4 \pi^{2} N_{c}\left(N_{c}+1\right)\left(2 N_{c}+1\right) /\left(6 N^{2}\right)$ and $N_{c}$ is the integer nearest to $N K_{c} a / 2 \pi$. Here we have used the fact that the Bloch wave vector $k$ takes the discrete values $2 n \pi / N a(n=1,2, \cdots N / 2)$. In this case, it then follows that for a fixed $t$,

$$
L_{c}(\lambda, t) \approx \exp \left(-\gamma t^{2}\right)
$$

when $\lambda \rightarrow \lambda_{c}=1$, where $\gamma=4 J^{2} \delta^{2} E\left(K_{c}\right) /(1-\lambda)^{2}$.

Notice that $L(\lambda, t)$ is less than $L_{c}(\lambda, t)$. So from the above heuristic analysis we may expect that, when $N$ is large enough and $\lambda$ is adjusted to the vicinity of the critical point $\lambda_{c}=1$, the LE will exceptionally vanish with time. On the other hand, we observe that $\gamma$ seems to approach zero in the thermodynamic limit $N \rightarrow \infty$ for $N a$ keeps as a constant in the process of taking this limit and $E\left(K_{c}\right) \propto 1 / N^{2}$. Since a true QPT can occur just in the thermodynamic limit, it is natural to doubt whether the QPT, and thus the induced decay of the LE, can happen at the critical point. In fact, due to the vanishing denominator $(1-\lambda)^{2}$ of $\gamma$ at the critical point of the QPT, the decay of the LE is still possible even for $\gamma$ having a vanishing numerator. For a practical system used to demonstrate the QPT induced decay of the LE, the particle number $N$ is large, but finite, and then the practical $\gamma$ does not vanish.

Now we resort to numerical calculation to test the heuristic analysis. For $N=50 \sim 250, \delta=0.1$, the LE are calculated numerically from the exact expression (8) with the parameters within the ranges $\lambda \in[0,2], t \in[0,27 / J]$. The results are demonstrated in Figs. 2a and $2 \mathrm{~b}$.

In Fig. 2a there exists a deep valley in the domain around the line $\lambda=\lambda_{c}-\delta=0.9$. This reflects the fact that near the critical point of the surrounding system the LE is very sensitive to the perturbation experienced by the surrounding system. At the critical point, with a highly enhanced decay of LE, the central system transits from a pure state to a mixed state due to its entanglement with the surrounding system. The five curves in Figure $2 \mathrm{~b}$ clearly demonstrate the influence of $N$ on the decay behavior of the LE. At $\lambda=\lambda_{c}-\delta=0.9$, the LE decays
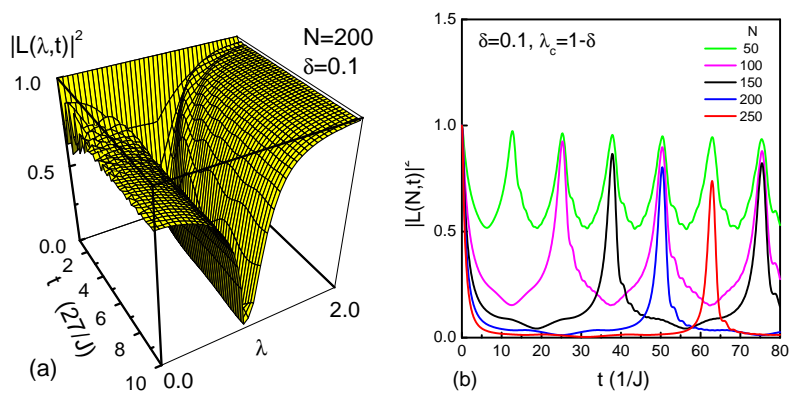

FIG. 2: (Color online) (a) Three dimensional (3-D) diagram of the LE $|L(\lambda, t)|^{2}$ as the function of $\lambda$ and $t$ for the system with $N=200$. The valley around the critical point $\lambda_{c}=1$ indicates that the decay of LE is enhanced by the QPT . The profile at $\lambda=1$ is in agreement with the analytical analysis. (b) The cross sections of the $3-\mathrm{D}$ surface for the systems of $N=50,100,150,200$, and 250 at $\lambda=\lambda_{c}-\delta=0.9$. It shows that the quasi-period of the LE is proportional to the size of the surrounding system .

and revives as time increases. The period of the revival of the LE is proportional to the size of the surrounding system.

Decays and revivals of Loschmidt echo as a witness of QPT: The novel phenomenon of the synchronization between the QPT and the enhanced decay of the LE mentioned above and its physical implication deserves further exploring. Generally, the two terms in $H_{e}$ represent two competitive physical effects with different order tendencies: in the weak coupling case $\lambda \ll 1$ the ground state is either all spins up or all spins down, while in the strong coupling case $\lambda \gg 1$ the ground state tends to the saturated ferromagnetic state with all the spins pointing right. When $\lambda$ takes the value of the order unity, the qualitative properties of the ground states for $\lambda>1$ and $\lambda<1$ are similar to those for $\lambda \gg 1$ and $\lambda \ll 1$ respectively. Only the critical point $\lambda=1$ has genuinely different properties.

The singular behavior of QPT at $\lambda=\lambda_{c}$ reflects the hypersensitivity of the ground states of the surrounding system with respect to the perturbation coupling imposed by the central system, which is reflected by the evolution of LE. We can thus expect quantum evolution of the surrounding system to inherit this sensitivity, which can also be understood as a signature of quantum chaos: For a quantum system prepared in the identical initial state, two slightly different interactions can lead to two quite different quantum evolutions. Mathematically speaking, this means the LE, initially equal to 1 , will decay with time and finally vanish. In this sense the sensitivity of quantum evolution to perturbation plays a crucial role in inducing the decay of LE. Due to the perturbations of two effective potentials caused by $|e\rangle$ and $|g\rangle$ respectively, the LE can decrease to zero due to the singularity at the critical point and the macroscopic enhancement of phase 

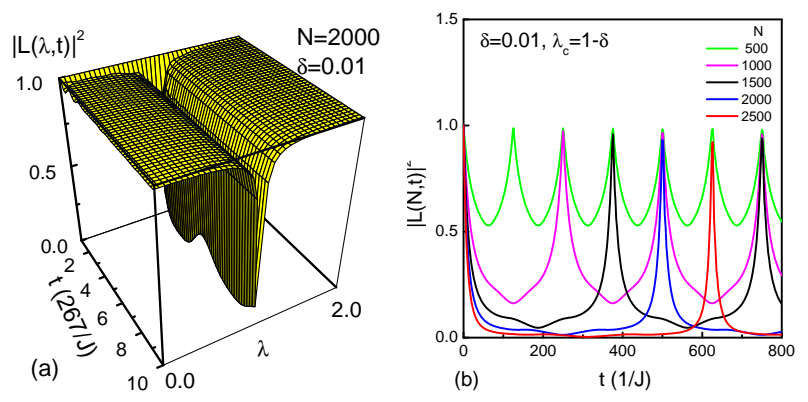

FIG. 3: (Color online) The quantum phase transition enhanced decay of LE at large $N$ limit for small $\delta$ : except for $\delta=0.01$ the explanations are the same as that in Fig. 2 .

randomness for large $N$, only at which QPT occurs 12 .

Next, we numerically investigate the LE for system with finite $N$. It turns out that as $N$ increases the LE will vanish for very small $\delta$. For example, we take $\delta=$ $0.01, N=500 \sim 2500$ and compare the numerical results illustrated in Fig. 3 with those for $\delta=0.1, N=50 \sim 250$ in Fig. 2. From Fig. 2a and 3a one can clearly see that the valley narrows as $\delta$ decreases and $N$ increases. On the other hand, the comparison between Fig. $2 \mathrm{~b}$ and $3 \mathrm{~b}$ shows an interesting phenomenon in the LE at the critical point $\lambda_{c}$. Firstly, the periods of the revival of the LE for the two cases are both proportional to the size of $E$. Secondly, the behaviors of the LE in Fig. 2b and $3 \mathrm{~b}$ are almost same. The numerical results seem to suggest an extrapolation for a scaling behavior, i.e., the LE at the critical point $L_{c}(t, \delta, N)$ is invariant under the scaling transformation $t \rightarrow t / \alpha, \delta \rightarrow \alpha \delta$, and $N \rightarrow$ $N / \alpha$. This prediction is in agreement with the analytical analysis with the small $J t$ approximations. Therefore, our result opens a possibility that the distinctive picture of the decays and revivals of the LE may serve as a good witness of QPT in the case of finite $N$.

Conclusion: In summary, by a special model, we have obtained the exact expression of the LE and analyzed the possible relation between the quantum-classical transition of the central system, characterized by the LE of the surrounding system, and the occurrence of a QPT in its surrounding system $E$. Both the heuristic analysis and the numerical calculations we performed reveal a novel mechanism of the decay of the LE. It is well known that quantum critical phenomenon is closely associated with the entanglement among the qubits consisting of the surrounding system [16]. We would like to stress that our present study is from a different perspective; our emphasis is on the relation between the QPT of the surrounding system and its entanglement with the central system $S$, which is qualitatively characterized by the LE. In our model, the maximal quantum entanglement between $S$ and $E$ can be reached and then the central system transits from a pure state to a mixed state when a QPT of $E$ takes place in one of the two evolution branches. This result suggests an unexplored and rather intriguing relationship among entanglement, LE, decoherence and criticality.

This work is supported by the NSFC with grant Nos. 90203018, 10474104 and 60433050, and NFRPC with Nos. 2001CB309310 and 2005CB724508. CPS thanks T. Xiang, L. Yu and Tien Kieu for helpful discussions.

* Electronic address: suncp@itp.ac.cn URL: http://www.itp.ac.cn/ ${ }^{\sim}$ suncp

[1] E. Joos, H.D. Zeh, C. Kiefer, D. Giulini, J. Kupsch and I-O Stamatescu, Decoherence and the Appearance of a Classical World in Quantum Theory, second edition, (Springer press, Berlin, 2003).

[2] W.H. Zurek, Phys. Today 44(10), 36(1991); S. Haroche, Phys. today, 51, 36 (1998).

[3] K. Hepp, Hev. Phys. Acta, 45, 237 (1972).

[4] J. S. Bell, Hev. Phys. Acta, 48, 93 (1975).

[5] H. Nakazato and S. Pascazio, Phys. Rev. Lett. 70, 1 (1993)

[6] M. Cini, Nuovo Cimento 73B, 27 (1983).

[7] C.P. Sun, Phys. Rev. A 48, 898 (1993), in QuantumClassical Correspondence, Ed. by D.H. Feng and B.L. Hu 99-106 (International Press,1994); in Quantum Coherence and Decoherence, Ed. by K. Fujikawa and Y.A. Ono, pp. 331-334, (Amsterdam: Elsevier Science Press, 1996); C.P. Sun, X.X. Yi and X.J. Liu, Fortschr. Phys. 43, 585 (1995).

[8] A. Peres, Quantum Theory: Concepts and Methods, (Kluwer Academic Publishers, Dordrecht, 1995).

[9] Z.P. Karkuszewski, C. Jarzynski, and W.H. Zurek, Phys. Rev. Lett. 89, 170405 (2002); F.M. Cucchietti, D.A.R. Dalvit, J.P. Paz and W.H. Zurek, ibid. 91, 210403 (2003); R. A.Jalabert and H. M. Pastawski, ibid. 86, 246 (2001).

[10] S. Sachdev, Quantum Phase Transition, (Cambridge University Press, Cambridge, 1999).

[11] C. Emary and T. Brandes, Phys. Rev. Lett. 90, 044101 (2003)

[12] P. Zhang, X.F. Liu and C.P. Sun, Phys. Rev. A 66, 042104 (2002).

[13] W.H. Zurek, U. Dorner and P. Zoller, Phys. Rev. Lett. 95, 105701 (2005).

[14] P. Pfeuty, Phys. Lett. A 72, 245 (1979).

[15] see the second reference in Ref. [9].

[16] G. Vidal, J.I. Latorre, E. Rico and A. Kitaev, Phys. Rev. Lett. 90, 227902 (2003); F. Verstraete, S. Guha, S. Lloyd, L. Maccone, J.H. Shapiro and H.P. Yuen, ibid. 92, 027901 (2004); L.-A. Wu, M.S. Sarandy and D.A. Lidar, ibid. 93, 250404 (2004); S.-J. Gu, S.-S. Deng Y.-Q. Li and H.-Q. Lin, ibid. 93, 086402 (2004); Y. Chen, P. Zanardi, Z.D. Wang and F.C. Zhang, quant-ph/0407228 X.-F. Qian, T. Shi, Y. Li, Z. Song and C.P. Sun, Phys. Rev. A 72, 012333 (2005).

[17] C.P. Sun, H. Zhan and X.F. Liu, Phys. Rev. A 58, 1810 (1998). 